\title{
Update on treatment modalities for optic nerve sheath meningiomas
}

\author{
James K. LiU, M.D., Scott Forman, M.D., ChitTi R. Moorthy, M.D., \\ AND DEBORAH L. BENZIL, M.D. \\ Department of Neurosurgery, University of Utah School of Medicine, Salt Lake City, Utah; and \\ Departments of Ophthalmology, Radiation Oncology, and Neurosurgery, New York Medical College, \\ Valhalla and New York, New York
}

\begin{abstract}
Optic nerve sheath meningiomas (ONSMs) represent 1 to $2 \%$ of all meningiomas and one third of all optic nerve tumors. The management of ONSMs is controversial. Traditional surgical removal often results in postoperative blindness in the affected eye and thus has been abandoned as a treatment option in most patients. Surgery may be unnecessarily aggressive, especially if the patient has useful vision. When these tumors are left untreated, however, ensuing progressive visual impairment may lead to complete blindness. More recently, radiotherapy has gained wider acceptance as a treatment for these lesions. The authors of some reports have suggested that fractionated stereotactic radiosurgery (SRS) may be the best option for treating primary ONSMs. In patients with documented progressive visual deterioration, fractionated SRS may be effective in improving or stabilizing remaining functional vision. The authors review the clinical presentation, radiographic characteristics, and management of ONSMs, emphasizing the use of fractionated SRS.
\end{abstract}

\section{KeY WORDS • optic nerve sheath meningioma $\bullet$ benign $\bullet$ stereotaxy - radiosurgery $\bullet$ dose fractionation $\bullet$ orbital neoplasm}

Meningiomas involving the optic nerve and the anterior visual pathways have intrigued neurosurgeons and ophthalmologists for nearly a century. The first definitive case of an ONSM was described in $1936 .{ }^{6}$ By 1938 , the potential had been demonstrated for removal of these tumors by complete excision of the optic nerve involving the tumor. ${ }^{7}$

Optic nerve sheath meningiomas account for 1 to $2 \%$ of all meningiomas, $1.7 \%$ among orbital tumors, and are the second most common optic nerve tumor after optic gliomas. ${ }^{7,9,15,30,37}$ These tumors typically affect middle-aged women and present with progressive visual loss. Most ONSMs are unilateral; only 5\% are bilateral. There is no strong evidence for right or left laterality. ${ }^{9}$

Optimal treatment of ONSMs remains undefined. ${ }^{12,15}$ Conservative treatment can be considered because of the indolent growth pattern of these tumors and the extremely low mortality rates associated with them. Over time, however, deterioration of vision always occurs. Traditional excision almost always results in postoperative

Abbreviations used in this paper: $\mathrm{CT}=$ computerized tomography; $\mathrm{MR}=$ magnetic resonance; ONSM = optic nerve sheath meningioma; SRS = stereotactic radiosurgery; $3 \mathrm{D}=$ three-dimensional. blindness to the affected eye because of the intimate circumferential relationship of the optic nerve and the central retinal artery. Although a few authors have reported rare cases of improved vision after resection, ${ }^{1,5,10,23}$ surgery offers no benefit to most patients with ONSMs and should be reserved for those in whom blindness, severe proptosis, or intracranial involvement has developed..$^{9,15}$

Radiotherapy of intracranial meningiomas has long been known to reduce recurrence and tumor progression..$^{17,18,35,36}$ In the last decade, conventional radiotherapy has been used to treat ONSM, and visual function has improved in $75 \%$ of cases. ${ }^{9,14,15,31}$ The complications of radiation-induced optic neuropathy and radiation necrosis, however, ${ }^{4,26,28}$ led to the search for better radiation-delivery techniques. The advent of 3D conformal radiotherapy and SRS has allowed administration of higher-dose radiation to a more specific target, sparing unnecessary radiation to neighboring structures. ${ }^{8,11,16,20}$ Stereotactic radiosurgery involves using a single, high dose of radiation, which may be toxic in the treatment of ONSMs. ${ }^{33}$ Fractionated SRS involves similar computer-image integration, but with a relocatable frame that facilitates a staged dosing scheme. This technique allows radiation to be administered with a high degree of accuracy at a dose-per- 
fraction known to be associated with a low risk of radiation-induced optic neuropathy. ${ }^{2,8,12,22,29}$

The authors of recent reports have demonstrated the potential efficacy of fractionated SRS in preserving or improving vision in patients who harbor ONSMs and who experience progressive visual loss. ${ }^{2,12,20,22,25}$ Known radiobiological concepts explain how a fractionated therapeutic regimen can arrest tumor growth while minimizing radiation-related toxicity to the optic nerve. We review the clinical presentation and radiographic/neuroimaging characteristics of ONSMs and discuss their management with fractionated SRS.

\section{CLINICAL PRESENTATION}

In a review of the literature, Dutton ${ }^{9}$ reported the mean age of patients with ONSMs at presentation to be 40.8 years (range 2.5-78 years) with a female predominance (61\%). Most ONSMs are unilateral; only $5 \%$ of cases are bilateral (Fig. 1).

The triad of progressive painless visual loss, optic nerve atrophy, and opticociliary shunt vessels is almost pathognomonic for the diagnosis of ONSM. ${ }^{32}$ The simultaneous manifestation of all three signs and symptoms, however, is rare. The most common presenting symptom is progressive loss of visual acuity or visual field (96\% of cases). Although long-term survival is likely, in most patients there is gradual progression to complete blindness in the involved eye if the tumor is left untreated. Transient visual obscurations, which may be gaze evoked or spontaneous, may precede, follow, or occur simultaneously with visual loss. ${ }^{30,38}$ Dyschromatopsia and afferent pupillary defects are other common manifestations of ONSMinduced optic neuropathy. The most common visual field defect is peripheral constriction., ${ }^{9,30}$ Other visual field defects, however, such as central or cecocentral scotoma, altitudinal defects, and enlargement of the blind spot, can manifest in compressive optic neuropathy. Patients with ONSM may also have optic disc abnormalities (98\%), proptosis (59\%), and opticociliary shunt vessels (30\%). ${ }^{9}$ Early stages of compressive neuropathy usually manifest as optic disc edema, which may eventually progress to optic atrophy. Opticociliary shunt vessels are a late sign of compressive optic neuropathy. They are presumed to be

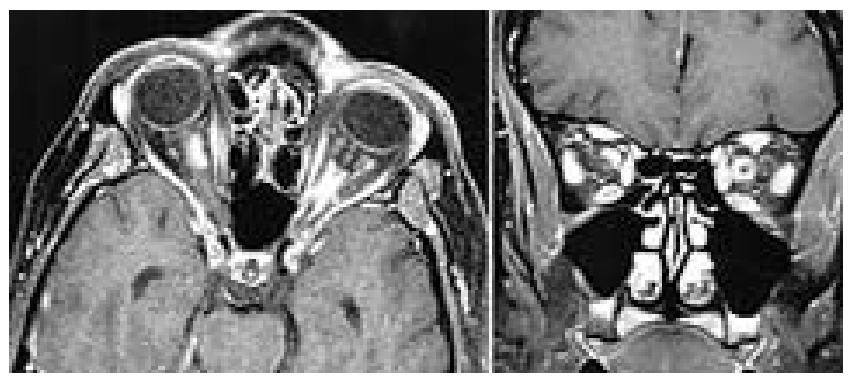

Fig. 1. Gadolinium-enhanced and fat-suppression MR images (axial [left] and coronal [right]) obtained in a patient with bilateral ONSMs. The left ONSM has enveloped the optic nerve circumferentially, and the characteristic doughnut sign can be observed. The right ONSM can be seen on the superomedial aspect of the right optic nerve. collateral vessels that develop between the retinal and choroidal venous circulation to allow blood to bypass the site of compression at the optic nerve. ${ }^{12,32}$

\section{RADIOGRAPHIC/NEUROIMAGING CHARACTERISTICS}

Before the age of CT scanning and MR imaging, the diagnosis of ONSM was difficult. Advances in neuroimaging have allowed the diagnosis of ONSM to be made on the basis of clinical and radiographic findings, thus obviating the need for obtaining a tissue biopsy sample. ${ }^{1,9,12,15,21}$ Early detection of ONSM is essential to prevent intracranial involvement, which may change the course of management.

On neuroimaging studies, ONSMs appear as a diffuse fusiform or tubular enlargement of the optic nerve. They are classified as intraorbital, intracanalicular (optic canal), or intraorbital with intracranial extension (Fig. 2). ${ }^{21}$ The tumor typically surrounds the optic nerve and results in a concentric thickening of the optic nerve diameter. Computerized tomography scans may demonstrate calcification of the nerve sheath or enlargement of the optic canal (Fig. 3). Axial CT scans reveal "tram tracking," a radiographic sign caused by the density difference between the thicker nerve sheath tumor and the residual optic nerve, which is characterized by central lucency (Fig. 3). ${ }^{9}$ On coronal CT scans, the tumor appears as a "doughnut," with the dense nerve sheath tumor encircling a central radiolucent optic nerve (Figs. 1-3). ${ }^{12}$ Gadolinium-enhanced and fat-suppression MR imaging can precisely

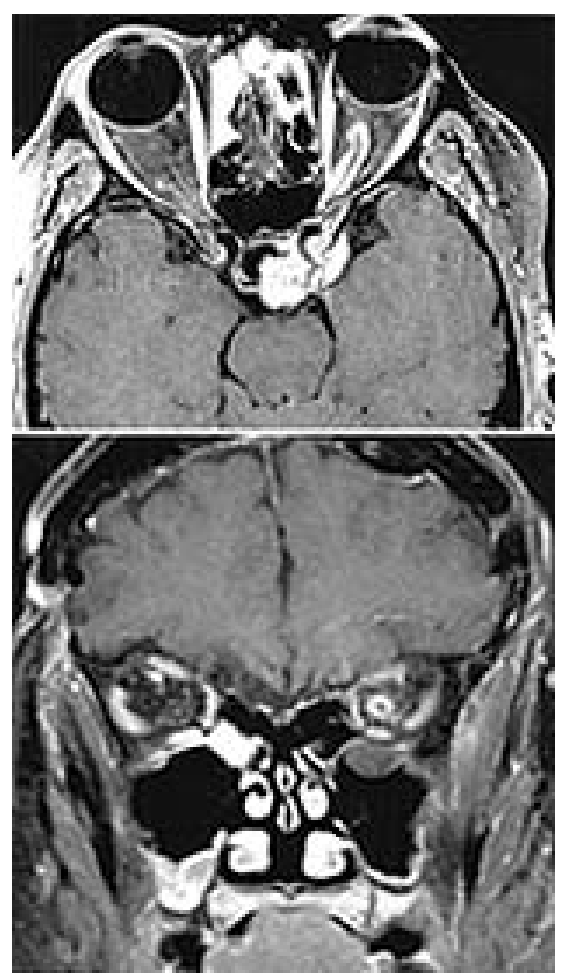

Fig. 2. Gadolinium-enhanced axial (upper) and coronal (lower) MR images demonstrating a left ONSM that extends intracranially into the cavernous sinus. 

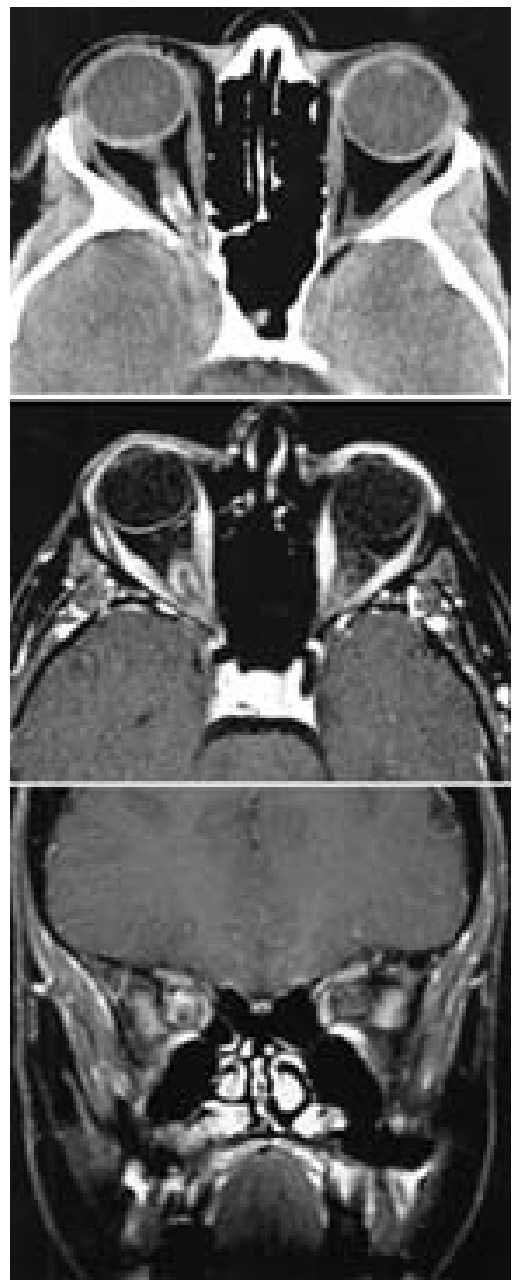

Fig. 3. Upper: Axial CT scan demonstrating calcification along the right optic nerve in a patient with an ONSM. Center and Lower: Axial (center) and coronal (lower) Gd-enhanced and fat-suppression MR images, demonstrating an ONSM arising from the right optic nerve. "Tram tracking" can be seen on the axial CT scan and MR image.

detect and demarcate ONSMs, especially those with an intracanalicular or intracranial component. ${ }^{21}$ The precision of imaging allows for great accuracy during treatment planning with fractionated SRS.

\section{MANAGEMENT: SURGERY AND RADIOTHERAPY}

\section{Surgical Management}

Cushing and Eisenhardt ${ }^{7}$ first described the resection of the entire optic nerve as a treatment for ONSMs in 1938. Surgical treatment became common, although continued attempts were made to resect the tumor while leaving the nerve intact. ${ }^{1,14,23,38}$

Attempts at excision of ONSMs may result in significant or total loss of remaining vision because most are located at or near the orbital apex and share their blood supply with the pia mater. ${ }^{13,15,16,38}$ En bloc resection in- volving removal of the optic nerve may be the best strategy in patients in whom functional vision is lost, especially if there is extensive intracranial extension. ${ }^{5,24}$ Resection may also play a role if the patient suffers from disfiguring proptosis and blindness. A craniotomy is performed to remove tumors that extend intracranially, and orbital exenteration is performed for orbital invasion beyond the optic nerve. ${ }^{1}$ The continued recommendation for resection in younger patients, in whom ONSMs may be more biologically aggressive, is highly controversial. These patients may be offered resection of both tumor and optic nerve from the chiasm to the globe, even when vision is functionally good. ${ }^{38}$ Rare cases of visual improvement after successful tumor resection have been reported. . $^{1,5,10,12,23}$

Optic nerve sheath meningiomas are not uniform in anatomy or presentation. The few that remain extradural, arising from intraorbital arachnoidal rests and secondarily involving the optic nerve,${ }^{24}$ can be removed without injury to the optic nerve. True primary ONSMs, in contrast, do not arise from the external surface of the nerve but rather from the area between the arachnoid and the dural sheaths of the optic nerve or from the intracranial cavity growing into this space. They usually wrap circumferentially around the optic nerve and extend posteriorly into the anulus of Zinn. Therefore, most ONSMs may not be completely resected without compromising the integrity of the optic nerve.

\section{Radiation Therapy}

Radiation therapy in the treatment of patients with ONSMs has gained wider acceptance in the past decade. ${ }^{4,9,18,31,34}$ Initial treatment with conventional radiotherapy resulted in visual improvement in nine of 12 patients. ${ }^{9,14,28,31}$ These techniques, however, expose the visual apparatus and neighboring tissues to large volumes of radiation, which may cause radiation necrosis and damage. ${ }^{4,18,28}$ Newer modalities, such as 3D conformal radiotherapy and fractionated SRS, use multiple beams of radiation to focus and shape accurately treatment to the lesion, thereby limiting exposure of adjacent normal tissue to damaging radiation. . $12,20,22,25$ It is known that the optic nerve tolerates a dose per fraction of $1.8 \mathrm{~Gy}$ to a total dose

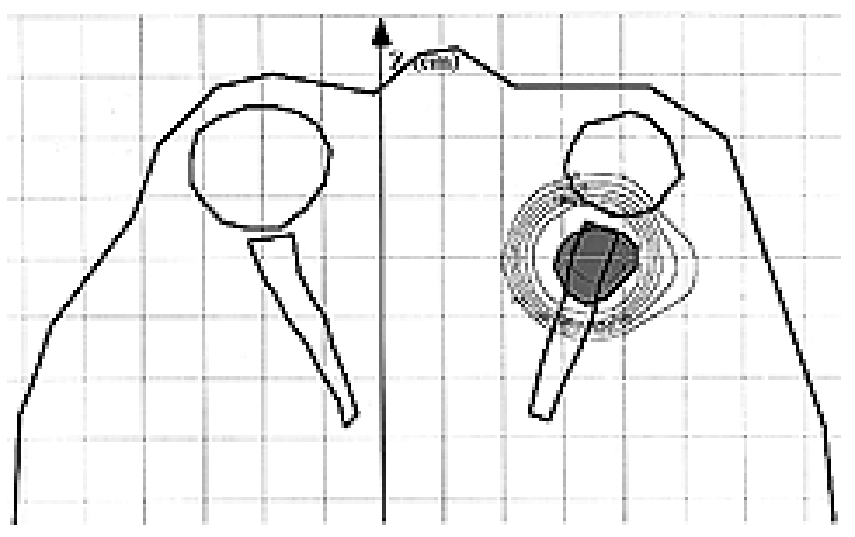

Fig. 4. Representative fractionated SRS dose plan. The tumor can be visualized in relation to the radiation volume and fall off. 
TABLE 1

Summary of data obtained in five patients with ONSMs

\begin{tabular}{|c|c|c|c|c|c|c|}
\hline $\begin{array}{l}\text { Case } \\
\text { No. }\end{array}$ & $\begin{array}{l}\text { Age (yrs), } \\
\text { Sex }\end{array}$ & $\begin{array}{l}\text { Affected } \\
\text { Side }\end{array}$ & Presentation & $\begin{array}{l}\text { Visual } \\
\text { Acuity }\end{array}$ & Outcome & $\begin{array}{l}\text { Follow-Up } \\
\text { Length (yrs) }\end{array}$ \\
\hline 1 & $63, \mathrm{~F}$ & lt & visual field \& acuity loss & $20 / 40$ & $\begin{array}{l}\text { 20/30, improved visual field, acuity \& } \\
\text { color vision }\end{array}$ & 3 \\
\hline 2 & $40, \mathrm{~F}$ & lt & visual loss, orbital pain, proptosis, headache & $20 / 30$ & 20/30, stable visual exam & 2 \\
\hline 3 & $50, \mathrm{~F}$ & lt & visual field loss & $20 / 20$ & 20/15, improved visual field \& acuity & 1 \\
\hline 4 & $60, \mathrm{M}$ & lt & visual field loss/change in color vision & $20 / 20$ & $20 / 20$, improved visual field \& color vision & 7 \\
\hline $5 *$ & $73, \mathrm{M}$ & bilat & blind (lt side), visual field \& acuity loss (rt side) & $20 / 40(\mathrm{rt})$ & 20/30 (rt), improved visual field \& acuity & 2 \\
\hline
\end{tabular}

* This patient presented with a left ONSM with intracranial extension and involvement of the right optic nerve. Because of the left eye blindness and remaining function in the right eye, fractionated SRS was performed to treat only the right eye.

of 54 Gy or a single dose of 6.5 to 8 Gy. ${ }^{19,26,33}$ Fractionated SRS maximizes the chance of vision preservation while minimizing the risk of radiation-induced optic neuropathy or optic neuritis (Fig. 4). Analysis of data gathered early in the use of fractionated SRS suggests that it effectively improves or stabilizes remaining functional vision in the subset of patients with ONSMs who present with documented progressive visual deterioration. ${ }^{2,3,12,20,22,25,27}$

In five patients harboring ONSMs, we administered fractionated SRS of 1.8-Gy fractions to a cumulative dose of 45 to $54 \mathrm{~Gy}$, without complications of delayed optic neuropathy (Table 1). ${ }^{22}$ All patients presented with progressive visual loss involving decreased visual fields, visual acuity, and color vision. We used a dedicated linear accelerator-based radiosurgical technique that involved a conventional fractionation protocol. Patients were fitted with the Gills-Thomas-Cosman relocatable frame (Radionics, Inc., Burlington, MA) and underwent stereotactic CT scanning and/or fat-suppression Gd-enhanced MR imaging. Treatment planning was performed using X-Knife 3D treatment planning software (Radionics). Treatments were planned to encompass the tumor at the $90 \%$ isodense line.

Within 3 months after treatment, four of the five patients experienced dramatic improvement in visual func-
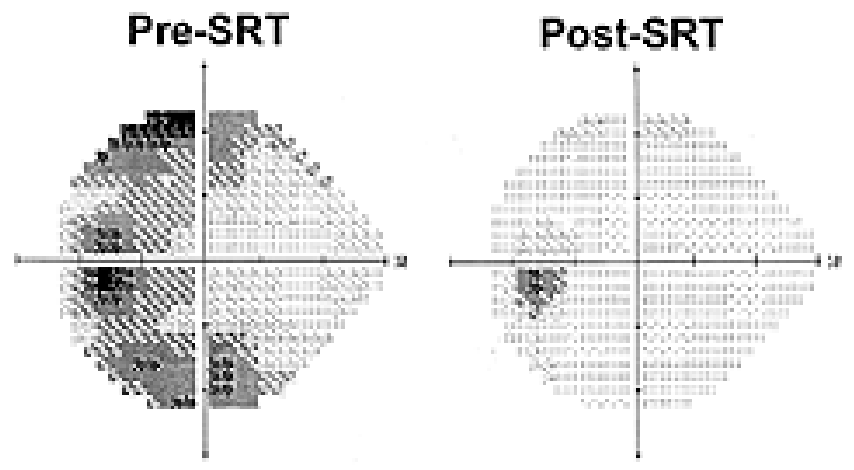

Fig. 5. Visual field testing results obtained in a 50-year-old woman with a left ONSM who presented with visual field loss. Prior to fractionated SRS treatment (Pre-SRT), the visual field test demonstrated diffuse and temporal depression of the left eye. After 30 1.8-Gy fractions (total dose 54 Gy) (Post-SRT) were delivered to the left ONSM, visual field testing showed dramatic improvement of the previous visual field defect. tion (Fig. 5). Visual field deficits resolved in all four, visual acuity improved in three, and color vision improved in two. Of the four patients whose visual field deficits resolved, one patient experienced resolution of opticociliary shunt vessels. Optic nerve atrophy and optic disc swelling, however, remained unchanged. In the fifth patient, stabilization of all visual functions occurred. Serial CT and MR images obtained during the follow-up periods revealed the size of the tumors in all five patients to be unchanged from baseline.

In another study, ${ }^{2} 33$ optic nerves in 30 patients with ONSMs were treated with a similar fractionated SRS protocol (1.8-Gy fractions to a total of 50 to $54 \mathrm{~Gy})$. Of the 24 eyes with preserved useful vision, 92\% demonstrated either stability $(50 \%)$ or improvement $(42 \%)$ in visual acuity and field testing. Tumor volume was reduced in four patients $(13 \%)$, and in the remainder there was no evidence of tumor progression or recurrence. Compared with patients who underwent observation, there was a $150 \%$ greater probability of visual improvement in the treatment group. In a third study, ${ }^{27} 15$ patients were treated and stable or improved vision occurred in all. Collectively, the use of fractionated SRS to treat patients with some residual vision has been successful in stabilizing (22 [47\%] of 47) or improving (23 [49\%] of 47) vision in nearly all cases (Table 2).

Fractionated SRS appears to confer tumor control and visual improvement or preservation of residual function. The risks of delayed optic neuropathy and secondary malignancies remain unknown, because there is limited longterm follow-up information. ${ }^{19,33}$ Fractionation of the dose and precise targeting, however, will likely reduce the risk of radiation injury of the optic nerve. Fractionated SRS may be the treatment option of choice for patients with ONSMs if vision preservation is the goal.

\section{CONCLUSIONS}

In patients harboring ONSM in whom functional vision remains, especially in the early stages of tumor growth, we recommend fractionated SRS to achieve vision stabilization or improvement and tumor control. Because meningiomas can recur slowly many years after excision, the true durable effects of fractionated SRS await longer follow-up study. Early visual improvement, however, should not be ignored even if visual symptoms recur or the tumor enlarges at a later date. Treatment with fractionated 
TABLE 2

Cumulative analysis of stereotactic radiosurgical treatment of ONSMs

\begin{tabular}{lcccccc}
\hline \hline & & & & & \multicolumn{2}{c}{ No. of Cases } \\
\cline { 5 - 7 } \multicolumn{1}{c}{ Authors \& Yr } & No. of & $\begin{array}{c}\text { Dose/ } \\
\text { Cases }\end{array}$ & $\begin{array}{c}\text { Traction (Gy) } \\
\text { Dose (Gy) }\end{array}$ & Improvement & Stabilization & Complications \\
\hline Lee, et al., 1996 & 1 & 1.8 & 50.4 & 1 & 0 & 0 \\
Fineman \& Augsburger, 1999 & 1 & 1.8 & 54 & 0 & 1 & 0 \\
Moyer, et al., 2000 & 1 & 1.8 & 50.4 & 1 & 0 & 0 \\
Andrews, et al., 2002 & 30 & 1.8 & $50-54$ & $10^{*}$ & $12^{*}$ & 4 \\
Liu, et al., 2002 & 5 & 1.8 & $45-54$ & 4 & 1 & 0 \\
Pitz, et al., 2002 & 15 & 1.8 & 54 & 7 & 23 & 0 \\
total & 53 & & & & 22 & 4 \\
\hline
\end{tabular}

* Of 24 eyes with preserved useful vision.

SRS does not limit later treatment options, as it might in other tumor entities, because complete optic nerve excision or orbital exenteration will likely be undertaken if surgery is performed. Stereotactic radiosurgery may also reduce the risk of radiation injury compared with conventional irradiation modalities.

\section{References}

1. Alper MG: Management of primary optic nerve meningiomas. Current status - therapy in controversy. J Clin Neuroophthalmol 1:101-117, 1981

2. Andrews DW, Faroozan R, Yang BP, et al: Fractionated stereotactic radiotherapy for the treatment of optic nerve sheath meningiomas: preliminary observations of 33 optic nerves in 30 patients with historical comparison to observation with or without prior surgery. Neurosurgery 51:890-904, 2002

3. Becker G, Jeremic B, Pitz S, et al: Stereotactic fractionated radiotherapy in patients with optic nerve sheath meningioma. Int J Radiat Oncol Biol Phys 54:1422-1429, 2002

4. Capo H, Kupersmith MJ: Efficacy and complications of radiotherapy of anterior visual pathway tumors. Neurol Clin 9: 179-203, 1991

5. Clark WC, Theofilos CS, Fleming JC: Primary optic nerve sheath meningiomas. Report of nine cases. J Neurosurg 70: 37-40, 1989

6. Coston TO: Primary tumor of the optic nerve, with report of a case. Arch Ophthalmol 15:696-702, 1936

7. Cushing H, Eisenhardt L: Meningiomas. Their Classification, Regional Behavior, Life History and Surgical Results. Springfield, IL: Charles C Thomas, 1938, pp 283-297

8. Das IJ, Downes MB, Corn BW, et al: Characteristics of a dedicated linear accelerator-based stereotactic radiosurgery-radiotherapy unit. Radiother Oncol 38:61-68, 1996

9. Dutton JJ: Optic nerve sheath meningiomas. Surv Ophthalmol 37:167-183, 1992

10. Ebers GC, Girvin JP, Canny CB: A 'possible' optic nerve meningioma. Arch Neurol 37:781-783, 1980

11. Eng TY, Albright NW, Kuwahara G, et al: Precision radiation therapy for optic nerve sheath meningiomas. Int J Radiat Oncol Biol Phys 22:1093-1098, 1992

12. Fineman MS, Augsburger JJ: A new approach to an old problem. Surv Ophthalmol 43:519-524, 1999

13. Hirst LW, Miller NR, Hodges FJ III, et al: Sphenoid pneumosinus dilatans: A sign of meningioma originating in the optic canal. Neuroradiology 22:207-210, 1982

14. Ito M, Ishizawa A, Miyaoka M, et al: Intraorbital meningiomas. Surgical management and role of radiation therapy. Surg Neurol 29:448-453, 1988
15. Kennerdell JS, Maroon JC, Malton M, et al: The management of optic nerve sheath meningiomas. Am J Ophthalmol 106: 450-457, 1988

16. Klink DF, Miller NR, Williams J: Preservation of residual vision 2 years after stereotactic radiosurgery for a presumed optic nerve sheath meningioma. J Neuroophthalmol 18: 117-120, 1998

17. Kondziolka D, Lunsford LD, Coffey RJ, et al: Stereotactic radiosurgery of meningiomas. J Neurosurg 74:552-559, 1991

18. Kupersmith MJ, Warren FA, Newall J, et al: Irradiation of meningiomas of the intracranial anterior visual pathway. Ann Neurol 21:131-137, 1987

19. Leber KA, Bergloff J, Pendl G: Dose-response tolerance of the visual pathways and cranial nerves of the cavernous sinus to stereotactic radiosurgery. J Neurosurg 88:43-50, 1998

20. Lee AG, Woo SY, Miller NR, et al: Improvement in visual function in an eye with a presumed optic nerve sheath meningioma after treatment with three-dimensional conformal radiation therapy. J Neuroophthalmol 16:247-251, 1996

21. Lindblom B, Truwit CL, Hoyt WF: Optic nerve sheath meningioma. Definition of intraorbital, intracanalicular, and intracranial components with magnetic resonance imaging. Ophthalmology 99:560-566, 1992

22. Liu JK, Forman S, Hershewe GL, et al: Optic nerve sheath meningiomas: visual improvement after stereotactic radiotherapy. Neurosurgery 50:950-957, 2002

23. Mark LE, Kennerdell JS, Maroon JC, et al: Microsurgical removal of a primary intraorbital meningioma. Am J Ophthalmol 86:704-709, 1978

24. Miller NR: Comment on optic nerve sheath meningiomas. Clinical manifestations. Ophthalmology 91:1324-1326, 1984

25. Moyer PD, Golnik KC, Breneman J: Treatment of optic nerve sheath meningioma with three-dimensional conformal radiation. Am J Ophthalmol 129:694-696, 2000

26. Parsons JT, Bova FJ, Fitzgerald CR, et al: Radiation optic neuropathy after megavoltage external-beam irradiation: analysis of time-dose factors. Int J Radiat Oncol Biol Phys 30: 755-763, 1994

27. Pitz S, Becker G, Schiefer U, et al: Stereotactic fractionated irradiation of optic nerve sheath meningioma: a new treatment alternative. Br J Ophthalmol 86:1265-1268, 2002

28. Sarkies NJ: Optic nerve sheath meningioma: diagnostic features and therapeutic alternatives. Eye 1:597-602, 1987

29. Shrieve DC, Tarbell NJ, Alexander E III, et al: Stereotactic radiotherapy: a technique for dose optimization and escalation for intracranial tumors. Acta Neurochir Suppl 62:118-123, 1994

30. Sibony PA, Krauss HR, Kennerdell JS, et al: Optic nerve sheath meningiomas. Clinical manifestations. Ophthalmology 91: 1313-1326, 1984

31. Smith JL, Vuksanovic MM, Yates BM, et al: Radiation therapy 
for primary optic nerve meningiomas. J Clin Neuroophthalmol 1:85-99, 1981

32. Spencer WH: Primary neoplasms of the optic nerve and its sheaths: clinical features and current concepts of pathogenetic mechanisms. Trans Am Ophthalmol Soc 70:490-528, 1972

33. Tishler RB, Loeffler JS, Lunsford LD, et al: Tolerance of cranial nerves of the cavernous sinus to radiosurgery. Int $\mathbf{J}$ Radiat Oncol Biol Phys 27:215-221, 1993

34. Turbin RE, Thompson CR, Kennerdell JS, et al: A long-term visual outcome comparison in patients with optic nerve sheath meningioma managed with observation, surgery, radiotherapy, or surgery and radiotherapy. Ophthalmology 109:890-900, 2002

35. Valentino V: Radiosurgery in cerebral tumors and AVM. Acta Neurochir Suppl 42:193-197, 1988
36. Wara WM, Sheline GE, Newman H, et al: Radiation therapy of meningiomas. Am J Roentgenol Radium Ther Nucl Med 123:453-458, 1975

37. Wright JE, Call NB, Liaricos S: Primary optic nerve meningioma. Br J Ophthalmol 64:553-558, 1980

38. Wright JE, McNab AA, McDonald WI: Primary optic nerve sheath meningioma. Br J Ophthalmol 73:960-966, 1989

Manuscript received March 14, 2003.

Accepted in final form April 14, 2003.

Address reprint requests to: Deborah L. Benzil, M.D., Department of Neurosurgery, New York Medical College, Munger Pavilion, Valhalla, New York 10595. email:deborah_benzil @nymc.edu. 